\title{
A Reset State Estimator Using an Accelerometer for Enhanced Motion Control With Sensor Quantization
}

\author{
Jinchuan Zheng and Minyue Fu, Fellow, IEEE
}

\begin{abstract}
Sensor quantization is a key factor that deteriorates the tracking performance of positioning systems with low-resolution optical encoders. This paper presents a method to improve the performance of such systems by merging an accelerometer of low cost. First, to reject the external disturbance, friction force and system perturbations, we design a disturbance observer (DOB) based on acceleration signals. Second, a reset kinematic state estimator (RKSE) is designed using acceleration signals to make the state estimate immune to both system perturbations and input disturbances. Third, a state feedback controller is designed based on the internal model principle (IMP) for accurate sinusoidal reference tracking. Simulations and experimental results are used to demonstrate the effectiveness of the proposed control method for tracking position reference commands and its robustness to system uncertainties.
\end{abstract}

Index Terms-Disturbance observer, high precision tracking control, reset state estimation, sensor quantization.

\section{INTRODUCTION}

$\mathbf{L}$ INEAR MOTORs (LMs) are used in a variety of industrial applications which include laser cutting machines, semiconductor wafer inspection, high-accuracy alignment, imaging and precision inspection and many more. In such applications, the overall motion control performance (e.g., high repeatability and accuracy) is required by the associated control system. This, in turn, depends on the accuracy and richness of the sensors that yield measurement signals used for feedback control.

Optical encoders are the most prevalent sensing devices that are typically integrated into an LM system to detect the position of a moving object. The encoder is based on evenly spaced divisions or line counts on a glass or metal disk, which is simple in construction and easy to manufacture. Position control of the drive can be realized by direct feedback of the encoder signal to the controller. In addition, velocity control is possible by estimating the velocity from the encoder position signal [1]. However, the interval of the divisions adversely leads to the resolution limitation in position measurement. When the encoder output is used as the feedback signal in a servo system, the encoder quantization noise will degrade the achievable position accuracy [2] and even cause self-sustained oscillations (i.e., limit cycles) [3]. Sensor quantization is a

Manuscript received June 06, 2008; revised September 08, 2008. Manuscript received in final form October 05, 2008. First published March 24, 2009; current version published December 23, 2009. Recommended by Associate Editor R. Landers.

The authors are with the School of Electrical Engineering and Computer Science, The University of Newcastle, Callaghan, NSW 2308, Australia (e-mail: jinchuan.zheng@newcastle.edu.au; minyue.fu@newcastle.edu.au).

Digital Object Identifier 10.1109/TCST.2009.2014467 source of typical measurement inaccuracy in motion control systems. From the cost and effectiveness point of view, designers generally need to select an encoder with resolution consistent with the required performance. In digital control systems, quantization may be created not only by the sensor itself but also by the digital-to-analog converter (DAC). In most cases, the quantization noise is ignored during control design process if it is substantially small compared to the system noise and the desired position accuracy.

Quantization is inherently a nonlinear feature. Often the time, quantization noises are described by a statistical model [4], [5]. In order to alleviate the sensor quantization noise, numerical algorithms that can be simply implemented on a digital signal processor (DSP) have been reported. For instance, Kalman filters have been employed to suppress the sensor quantization noise under the assumption that they can be modeled as a white Gaussian noise [6], [7]. However, in motion control systems, signals tend to be more deterministic and exhibit stronger correlation over time. Thus, quantization noises behave as highly colored noises, which makes the Kalman filtering approach inferior. For this reason, there has been intense research on nonlinear filters such as extended Kalman filters [8], unscented Kalman filters [9] and particle filters [10], [11] attempting to improve estimation accuracy for non-Gaussian/nonlinear processes. The key practical issue in nonlinear filtering is real-time computational complexity [12], which generally limits its applications to motion control systems that require high servo bandwidth and thus are sensitive to computing cost. Alternatively, state estimators based on the observer theory have been extensively studied in [13]-[16], in which extra useful information is extracted from the quantizer model and then used to enhance the estimation. This paper presents a solution to this problem along this line by resetting the estimated state based on additional quantization information. In contrast to the approach above, the reset state estimator is relatively easy to design and adds insignificant computational cost.

In the presence of plant uncertainties and tracking trajectory variations, the performance of the state estimator becomes unreliable due to the nonlinear dynamics of the quantization noise. Replacing the sensor with a higher-resolution one would mean extra costs. A cost-effective way to improve the performance is to incorporate acceleration sensing. There are two main reasons for this option. First, the performance of linear accelerometers have recently improved a lot while the cost and size are both reduced significantly due to mass fabrication and the microelectro-mechanical systems (MEMS) technology. Second, the use of accelerometers has been well demonstrated in the literature (e.g., [17]-[21]) in improving motion control perfor- 
mance. For example, in state estimation, the benefit of acceleration sensing is yielding a kinematic model from the acceleration to the position, which is independent of the plant parameters [20]. Accordingly, a kinematic Kalman filter can be set up by using the acceleration measurement as an input for velocity estimation [21].

In this paper, we consider an LM control system integrated with a low-resolution position encoder and augmented by an accelerometer. The accelerometer is used to improve the position tracking control in two ways. One is in the design of a disturbance observer (DOB), the roles of which are for disturbance and friction compensation and for overcoming payload variations. It will be shown that the performance of a DOB with an acceleration signal as the input outperforms that with quantized position measurements because the acceleration sensing noise is relatively low compared to the quantization noise. The other is in the design of a reset kinematic state estimator (RKSE). It will be shown that the kinematic state estimator is more or less independent of the system model, thus, is not only robust against plant parameters but also insensitive to the input disturbance and friction force. Moreover, we attempt to further decrease the estimation error by extracting additional information from the quantized output, which is then used to update the estimated state by a reset technique. Previous research has demonstrated that the feedback controller with properly designed state resetting can outperform a purely linear controller (e.g., see [22] and [23]). Here, we will employ a reset technique to improve the state estimator. Our reset scheme is guided by the idea that the actual position is known exactly at the mid-point of two consecutive quantizer levels when the quantized measurement changes the quantization level. Finally, the estimated state is fedback to a controller derived based on the well-known internal model principle (IMP) design technique [24]. The IMP-based controller is simple to design and straightforward to implement. Further, it can track sinusoidal reference input with zero steady-state error if accurate state feedback is available.

The rest of this paper is organized as follows. Section II describes the LM plant model and the details of the sensors. Section III illustrates the fusion of the acceleration measurement and quantized position as feedback signals to a control structure which is comprised of three components, i.e., the DOB, the RKSE, and a state feedback controller. Details of these components are then presented in Sections IV-VI, respectively. Section VII presents experimental results to demonstrate the effectiveness of using the accelerometer and the RKSE in tracking control. Finally, conclusions are given in Section VIII.

Partial and related results of this paper were presented previously in [25].

\section{Plant Modeling}

The experimental setup for a linear motor positioning system (by Baldor Electric Company) is shown in Fig. 1. The linear motor has a $0.5 \mathrm{~m}$ travel range with a mounted optical encoder (by Renishaw PLC), and a voltage-to-current power amplifier. A simplified plant model of the linear motor system is described in Fig. 2, where the symbol definitions are described in Table I. The physical parameters of the linear motor are also listed in Table II, which are either provided by the manufacturer or identified from

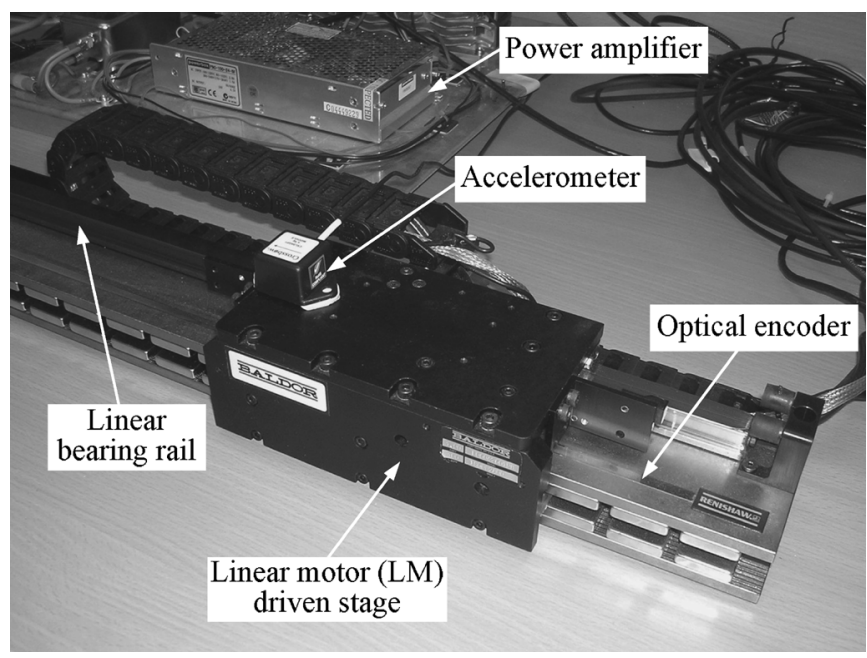

Fig. 1. Experimental setup of a linear motor positioning system.

experiments. In Fig. 2, $\Delta_{m}$ denotes gain variation mainly caused by various load mass, which is assumed to be slow-varying. The power amplifier dynamics is simplified as a constant gain $k_{f}$ as it has much higher bandwidth than the desired closed-loop servo bandwidth. We employ a nonlinear friction model in [3] to represent the friction model $g(v)$ in the linear motor system, which is given by

$$
g(v)=k_{v f} v+k_{c f} \operatorname{sgn}(v)+k_{s f} \operatorname{sgn}(v) e^{-|v / \sigma|}+\Delta_{f}
$$

where $\operatorname{sgn}(\cdot)$ denotes the sign function, $\Delta_{f}$ represents the unmodeled friction force, and other friction parameters are described in Table II, whose values are obtained by using the experimental method in [26]. Fig. 3 shows the measured and identified friction model, which verifies the accuracy of the identified friction model. Therefore, the identified friction model in (1) is used for the simulation of the actual linear motor position control performance.

\section{A. Position Sensing}

In our setup, we consider the position measured by an encoder. Thus, the measured position for control is a quantized version of the actual position given by

$$
y_{q}(t)=Q(y(t)) \text {. }
$$

The function $Q(\cdot)$ represents a uniform quantizer defined by

$$
Q(y)=i \cdot \Delta, \quad \text { if } y \in[(i-0.5) \Delta,(i+0.5) \Delta]
$$

where $i \in \mathbb{Z}$ and $\Delta>0$ denotes the constant quantization step size, which is also referred to as sensor resolution in motion control systems. We assume that the quantization range is infinite. Fig. 4 shows the input-output relationship of (3). The quantizer introduces a sensing error on the controlled output $y(t)$ as follows:

$$
\varepsilon(t)=y(t)-y_{q}(t)
$$

with

$$
|\varepsilon(t)| \leq \frac{\Delta}{2} \quad \forall t
$$




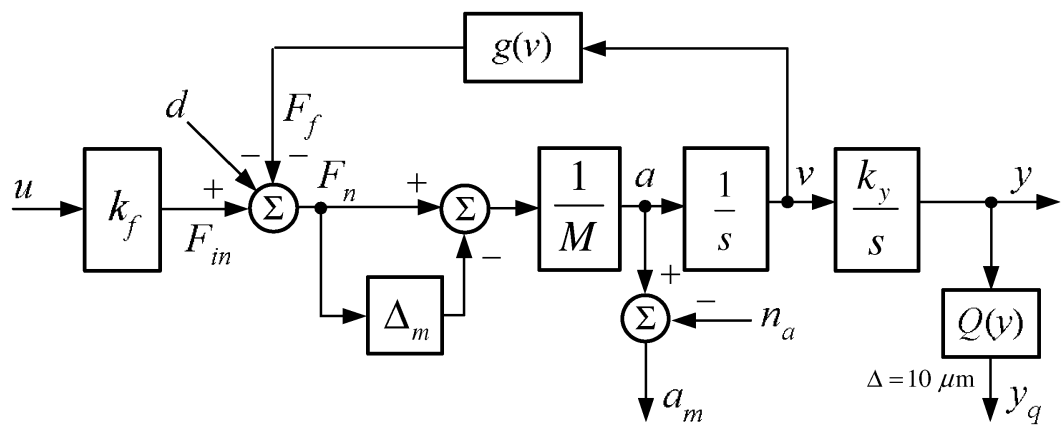

Fig. 2. Plant model of the linear motor positioning system (see Tables I and II for symbol definitions and parameter values).

TABLE I

SYMBOL DEFINITIONS IN PLANT MODEL

\begin{tabular}{llc}
\hline Symbol & Description & Unit \\
\hline$F_{i n}$ & applied input force & $N$ \\
$F_{n}$ & net input force causing acceleration & $N$ \\
$F_{f}$ & friction force & $N$ \\
$d$ & external force disturbance & $N$ \\
$u$ & applied control input & $V$ \\
$a$ & acceleration & $\mathrm{m} / \mathrm{s}^{2}$ \\
$v$ & velocity & $\mathrm{m} / \mathrm{s}$ \\
$y$ & position & $\mu \mathrm{m}$ \\
$a_{m}$ & measured acceleration & $\mathrm{m} / \mathrm{s}^{2}$ \\
$n_{a}$ & accelerometer noise & $\mathrm{m} / \mathrm{s}^{2}$ \\
$y_{q}$ & measured quantized position & $\mu \mathrm{m}$ \\
$g(v)$ & friction model & \\
$Q(y)$ & position encoder model & \\
\hline
\end{tabular}

TABLE II

PLANT MODEL PARAMETERS

\begin{tabular}{llcl}
\hline Parameter & Description & Value & Unit \\
\hline$k_{f}$ & voltage-to-force constant & 50 & $\mathrm{~N} / \mathrm{V}$ \\
$k_{y}$ & position sensor gain & $10^{6}$ & $\mu \mathrm{m} / \mathrm{m}$ \\
$M$ & moving mass of linear motor stage & 3.31 & $\mathrm{~kg}$ \\
$\Delta_{m}$ & gain variation due to load mass & {$\left[\begin{array}{lll}0 & 1\end{array}\right)$} & \\
$\Delta$ & position encoder resolution & 10 & $\mu \mathrm{m}$ \\
\hline$k_{v f}$ & viscous friction coefficient & 8.6 & $\mathrm{Ns} / \mathrm{m}$ \\
$k_{c f}$ & Coulomb friction level & 11.5 & $\mathrm{~N}$ \\
$k_{s f}$ & static friction level & 3.3 & $\mathrm{~N}$ \\
$\sigma$ & static friction velocity constant & 0.023 & $\mathrm{~m} / \mathrm{s}$ \\
\hline
\end{tabular}

The optical encoder in our setup has a resolution of $1 \mu \mathrm{m}$. But we pass the output through an artifical quantizer of (3) implemented in the DSP to simulate a lower resolution encoder by setting the quantizer step size

$$
\Delta=10 \mu \mathrm{m} .
$$

Under such a setting, the original output of the optical encoder is approximated as the actual position $y$ and used for monitoring

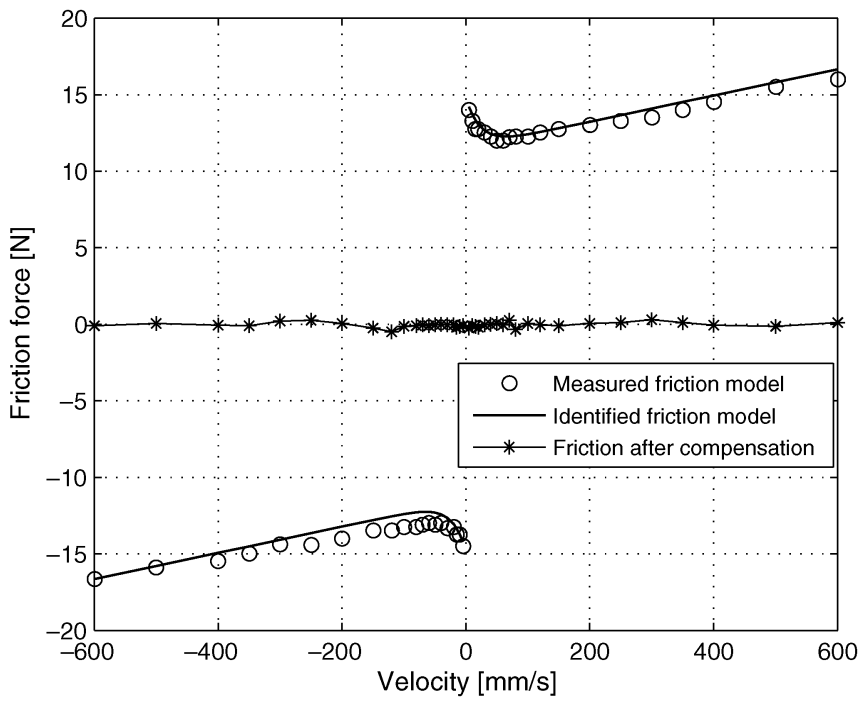

Fig. 3. Experimental friction model. (The vertical axis is measured in terms of the steady-state force input $F_{\text {in }}$ that compensates for the friction force to make the linear motor move at the corresponding constant velocity.)

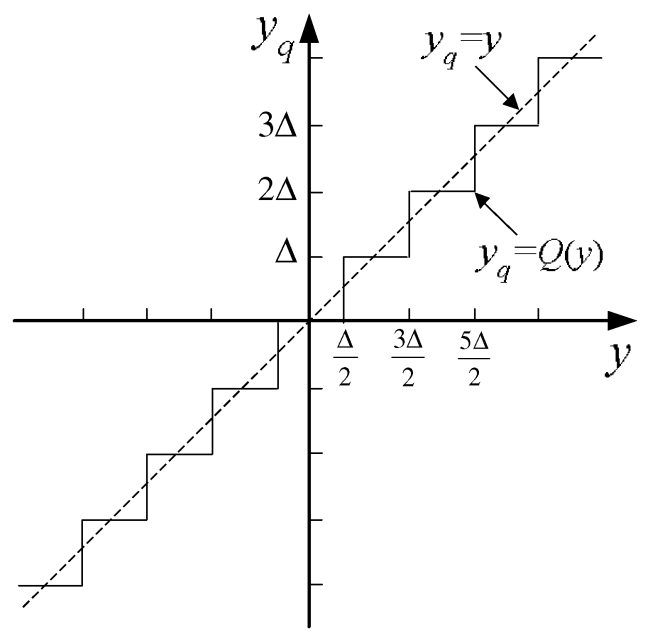

Fig. 4. Quantization characteristic; $\Delta$ : quantization step size (encoder resolution).

only, while the output of the artifical quantizer is used for control purposes. 

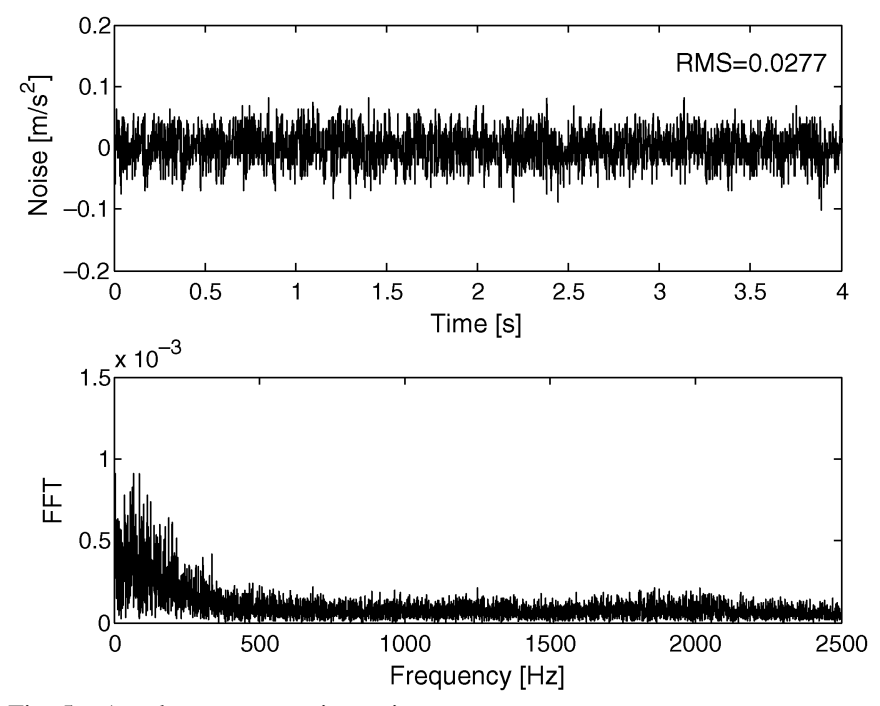

Fig. 5. Accelerometer sensing noise.

\section{B. Acceleration Sensing}

The accelerometer (by Crossbow Inc.) is mounted onto the linear motor and aligned to the linear bearing rail as shown in Fig. 1. This is used to measure the linear acceleration of the motor stage. The accelerometer offers a $\pm 39.2 \mathrm{~m} / \mathrm{s}^{2}$ measurement range, has a DC-100 Hz frequency bandwidth, and yields an analog voltage output proportional to the acceleration such that a direct interface to an analog-to-digital converter (ADC) for control purpose can be easily performed. The accelerometer after calibration offers a low-level sensing noise for our application, which is measured and shown in Fig. 5. We can see from the fast Fourier transform (FFT) spectrum that the noise behaves like white noise with higher densities in low frequencies. The accelerometer utilizes a silicon micro-machined technology that offers a compact sensor size with a significantly lower cost than a high-resolution optical encoder.

In the rest of this paper, we attempt to investigate how to fuse the measured acceleration signal and the quantized position signal for enhanced linear motor tracking performance. As such, a cost-effective method for linear motor positioning control can be obtained.

\section{Control Structure}

Our objective is to design a control law to make the position output of the linear motor stage accurately follow a sinusoidal reference input under the constraints of friction, load mass variations and the position quantization noise. This control task can be completed through a sequential design procedure. Fig. 6 shows a block diagram of the overall linear motor position control structure, where the controller is comprised of three components: the DOB, the RKSE, and the state feedback controller based on the IMP. The sequential design procedure simplifies the design into separate steps, each aiming for a specific goal and as such to achieve the overall control objective. Here, the DOB is first designed to compensate for the external disturbance, friction, and load mass variation. The proposed RKSE aims to suppress the quantization noise and thus to obtain the accurate position and velocity information, which is then used as the input to the state feedback controller for position tracking.

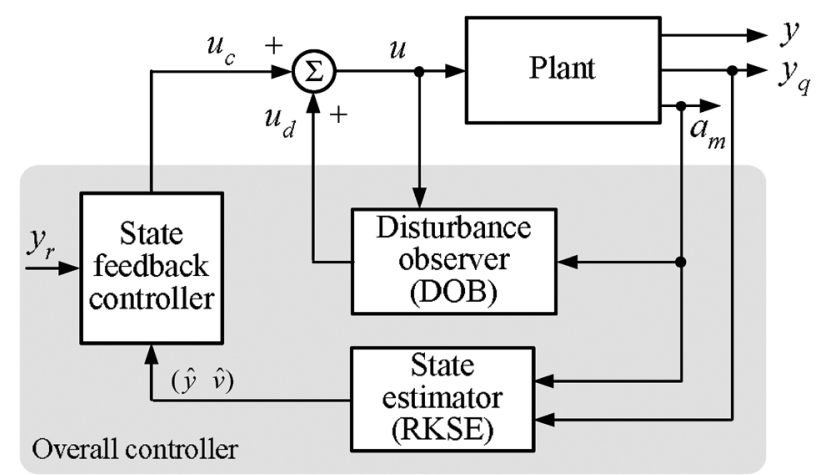

Fig. 6. Overall position control structure for the linear motor positioning system.

In what follows, we will present the design for each controller component, the effectiveness of which is then verified through simulations and experiments.

\section{Disturbance ObSERVER (DOB)}

The DOB technique and its variations [27] have been widely used in many servo systems and industrial applications. In hard disk drive servo systems, the DOB has been combined to compensate for pivot friction nonlinearity [18], reject shock and vibration disturbances [28], and to cancel the effects of modeling errors [29]. The error-based DOB is attempted to enhance the robustness and disturbance rejection for optical disk drive systems [30]. In [31], the DOB is implemented in a robot for force control instead of using the force sensor. More recently, communication disturbance observer is also proposed for time-delay compensation for network-based teleoperation control systems [32]. In this section, we apply the DOB technique incorporating the acceleration measurement for disturbance and friction compensation and for overcoming payload variations in the LM system.

From the plant model in Fig. 2, the friction force $F_{f}$, input disturbance $d$, and the gain uncertainty $\Delta_{m}$ exert adverse effects on the tracking performance. The role of DOB is to estimate the disturbance and thereby eliminate it. Further, the DOB observes and compensates for the input-output model discrepancies between the actual plant model and a nominal plant model embedded in the DOB. In our setup, the model discrepancies are mainly caused by the nonlinear friction and gain variations in the payload.

Fig. 7 shows the control structure of the DOB based on acceleration feedback. The reason for employing acceleration instead of quantized position feedback is to avoid introducing the quantization noise into the DOB loop. In addition, the DOB is easy to design and implement since the dynamics from the control input to the acceleration output can be approximated by a pure gain within the frequency bandwidth of our interest.

From Fig. 7, the sensed signals $u$ and $a_{m}$ go into the DOB and the output signal $u_{d}$ from the low-pass filter $F_{d}(s)$ is fed back to the control input. The dynamics from $u_{c}$ to the actual acceleration $a$ is then given by

$a=\frac{1-\Delta_{m}}{1-\Delta_{m} k_{f} F_{d}} \cdot\left(\frac{k_{f}}{M} \cdot u_{c}+k_{f} F_{d} \cdot n_{a}+\frac{k_{f} F_{d}-1}{M} \cdot\left(d+F_{f}\right)\right)$ 


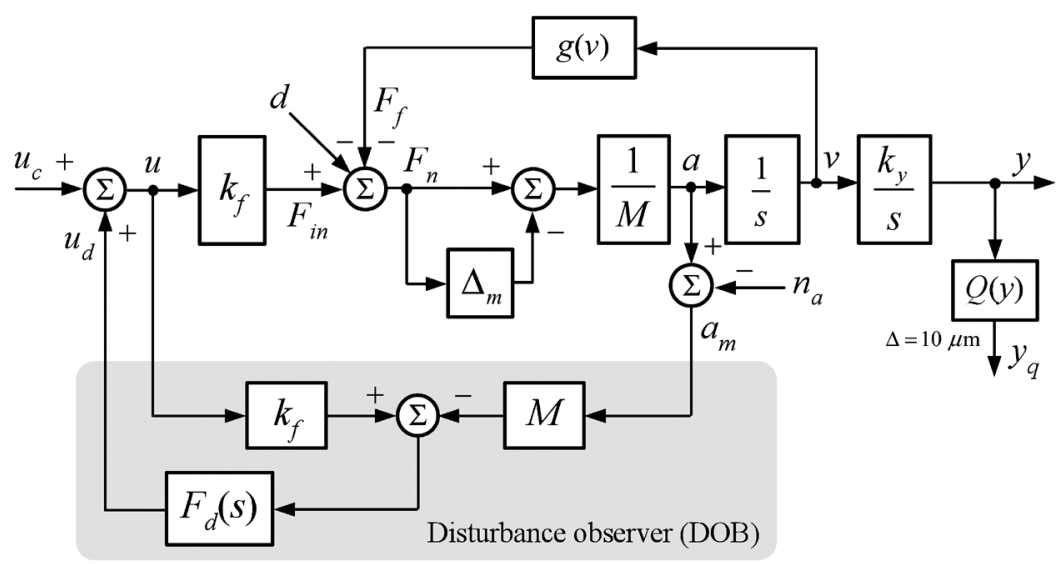

Fig. 7. Control structure of DOB based on acceleration sensing.

Ideally, given that $F_{d}(s) \equiv 1 / k_{f}$, we have

$$
a=\frac{k_{f}}{M} \cdot u_{c}+n_{a}
$$

Thus, the external disturbance and friction force are perfectly canceled, although the measurement noise is all-pass. This again explains the reason for using the acceleration signal as the input instead of the quantized position that contains a much higher level of noise. Practically, $F_{d}(s)$ is selected to be a low-pass filter to balance the compensation of sensing noise and disturbance. Since the nominal model from $u_{c}$ to $a$ can be treated as a constant gain, there is no strict requirement of the order of the filter $F_{d}$ for causality. One simple choice of $F_{d}(s)$ can be

$$
F_{d}(s)=\frac{1}{\tau s+k_{f}}
$$

where $\tau$ is a positive time constant chosen as 5 to 10 times the servo bandwidth such that the filter can be approximated as $F_{d} \approx 1 / k_{f}$ within the frequency bandwidth of interest. When the filter $F_{d}$ is applied, the input-output transfer function of the DOB is given by

$$
\begin{array}{r}
a=\frac{1-\Delta_{m}}{\tau s+k_{f}\left(1-\Delta_{m}\right)}\left(\left(\tau s+k_{f}\right) \frac{k_{f}}{M} \cdot u_{c}+k_{f} \cdot n_{a}\right. \\
\left.-\frac{\tau s}{M} \cdot\left(d+F_{f}\right)\right) .
\end{array}
$$

It is clear that the DOB (9) is stable provided that $\Delta_{m}<1$. Therefore, there exists an $\bar{\omega}>0$ such that

$$
a=\frac{k_{f}}{M} \cdot u_{c}+\omega, \quad|\omega|<\bar{\omega}
$$

where $\omega$ represents an equivalent residual disturbance that is beyond the capability of the DOB to reject. Generally, $\omega$ contains high frequency components, which can be further filtered by the low-pass featured plant model.

Fig. 3 shows the effectiveness of the DOB on friction compensation by experiments. Accordingly, the linear motor plant model behaves as a second-order linear system given by

$$
P_{n}(s)=\frac{y(s)}{u_{c}(s)}=\frac{k_{f} k_{y}}{M} \cdot \frac{1}{s^{2}}
$$

which is employed as the nominal model for the design of the state feedback controller in Section VI.

\section{Reset Kinematic State Estimator (RKSE)}

In this section, we will employ both the quantized position signal and the acceleration signal to design the state estimator. Since acceleration and velocity are the two principal quantities which describe how the position changes, a purely kinematic model that relates the acceleration $a(t)$ to the position $y(t)$ is given by

$$
\frac{d^{2} y(t)}{d t^{2}}=k_{y} a(t)
$$

However, in practical applications, only the measured kinematic variables $a_{m}$ and $y_{q}$ are available, which may deviate from their actual values. Further, the initial values of the kinematic variables are generally unknown. Thus, it is essential to build a closed-loop estimator to reduce the sensing noise without the knowledge of the initial conditions. To proceed, we represent the kinematic model in the state space as follows:

$$
\begin{aligned}
\dot{x}(t) & =A_{r} x(t)+B_{r} a(t) \\
x(0) & =x_{0} \\
y(t) & =C_{r} x(t) \\
y_{q}(t) & =C_{r} x(t)-\varepsilon(t) \\
a_{m}(t) & =a(t)-n_{a}(t)
\end{aligned}
$$

where

$$
x=\left[\begin{array}{l}
y \\
v
\end{array}\right], \quad A_{r}=\left[\begin{array}{cc}
0 & k_{y} \\
0 & 0
\end{array}\right], \quad B_{r}=\left[\begin{array}{l}
0 \\
1
\end{array}\right], C_{r}=\left[\begin{array}{ll}
1 & 0
\end{array}\right] .
$$

Note that the acceleration is treated as the input, and the system matrices involve none of the plant parameters. Thus, It is clear that the kinematic model has advantages: It is a simple and exact representation of the system state up to certain bandwidth. It involves neither physical parameters nor external disturbance, friction, and model uncertainties.

In order to achieve a smooth and accurate estimate of the state using the measured acceleration and the quantized position, the 
most common way is to use the standard state estimator (SSE) as follows:

$$
\begin{aligned}
& \dot{\hat{x}}(t)=A_{r} \hat{x}(t)+B_{r} a_{m}(t)+L\left(y_{q}(t)-\hat{y}(t)\right) \\
& \hat{y}(t)=C_{r} \hat{x}(t)
\end{aligned}
$$

where $\hat{x}$ and $\hat{y}$ are, respectively, the estimate of the state and controlled output, and $L \in \mathbb{R}^{2 \times 1}$ is the estimator gain. Conventionally, $L$ can be artificially selected by using the pole placement method or optimally designed using the Kalman filtering technique, where both the process noise and quantization error are assumed to be white Gaussian noises. However, more accurate state estimate is possible if the quantization scheme is further employed.

Though the quantized output $y_{q}$ gives discretized measurements of the actual $y$, we can still extract additional information given a sequence of measured quantized outputs. From Fig. 4, we can obtain the following observations.

1) At the time when the quantized output transits from one quantization step to another, the actual position is known exactly, which is at the midpoint of the two consecutive quantization levels.

2) At the time when the quantizer holds its output equivalent to a certain quantization step, according to the fact of (5), the actual position relative to the quantized output is always bounded by $\Delta / 2$. This implies that any estimate of $y$ at these times must be bounded by $\Delta / 2$ relative to the instantaneous quantized output.

We note that these observations can be used to improve the estimate of the state.

In the following, we present a RKSE with the use of a reset technique.

\section{A. Reset Kinematic State Estimator}

First, we start with the standard state estimator (15), (16), where $L$ is chosen such that $A_{r}-L C_{r}$ is stable. We then introduce a vector $H$ as follows [15]:

$$
H=P^{-1} C_{r}^{T}\left(C_{r} P^{-1} C_{r}^{T}\right)^{-1}
$$

where $P \in \mathbb{R}^{2 \times 2}$ is a positive definite symmetric matrix, which is the solution of the following Lyapunov function:

$$
\left(A_{r}-L C_{r}\right)^{T} P+P\left(A_{r}-L C_{r}\right)+I=0 .
$$

Next, we modify the standard state estimator $(15),(16)$ to incorporate the additional information from the quantized output. Namely, we reset the estimated state in the following two cases.

1) At the reset time $t_{k, 1}$, which is defined as

$$
t_{k, 1}: y_{q}\left(t_{k, 1}\right) \neq y_{q}\left(t_{k, 1}^{-}\right)
$$

the estimated state is reset by

$$
\hat{x}\left(t_{k, 1}\right)=\hat{x}\left(t_{k, 1}^{-}\right)-H\left(\hat{y}\left(t_{k, 1}^{-}\right)-\frac{1}{2}\left(y_{q}\left(t_{k, 1}\right)+y_{q}\left(t_{k, 1}^{-}\right)\right) .\right.
$$

It is easy to verify that the new estimated state leads to

$$
\begin{aligned}
\hat{y}\left(t_{k, 1}\right) & =\frac{1}{2}\left(y_{q}\left(t_{k, 1}\right)+y_{q}\left(t_{k, 1}^{-}\right)\right) \\
& =y\left(t_{k, 1}\right) .
\end{aligned}
$$

2) At the prespecified reset time $t_{k, 2}$ defined by

$$
t_{k, 2}=k T, \quad k \in \mathbb{Z}^{+}
$$

where $T$ indicates a predefined reset interval (e.g., equivalent to the sampling period), the estimated state is then reset by

$$
\begin{aligned}
\hat{x}\left(t_{k, 2}\right)=\hat{x}\left(t_{k, 2}^{-}\right)-H & \left(\hat{y}\left(t_{k, 2}^{-}\right)-y_{q}\left(t_{k, 2}\right)\right. \\
& \left.-\operatorname{Sat}_{\frac{\Delta}{2}}\left(\hat{y}\left(t_{k, 2}^{-}\right)-y_{q}\left(t_{k, 2}\right)\right)\right)
\end{aligned}
$$

where $\operatorname{Sat}(\cdot)$ is the saturation function with the saturation level of $\Delta / 2$. We can see that the new estimated state can lead to (24) shown at the bottom of the page. Hence, it can be seen that overestimation of the estimated output $\hat{y}$ is prevented while the estimated output is unchanged if overestimation is not detected.

By incorporating the preceding reset schemes into the SSE (15), we obtain a RKSE, which has the form

$$
\begin{aligned}
\dot{\hat{x}}(t)= & A_{r} \hat{x}(t)+B_{r} u(t)+L\left(y_{q}(t)-\hat{y}(t)\right) \\
& t \notin\left\{t_{k, 1} ; t_{k, 2}\right\} \\
\hat{x}\left(t_{k, 1}\right)= & \hat{x}\left(t_{k, 1}^{-}\right) \\
& -H\left(\hat{y}\left(t_{k, 1}^{-}\right)-\frac{1}{2}\left(y_{q}\left(t_{k, 1}\right)+y_{q}\left(t_{k, 1}^{-}\right)\right)\right. \\
& t_{k, 1}: y_{q}\left(t_{k, 1}\right) \neq y_{q}\left(t_{k, 1}^{-}\right) ; \\
\hat{x}\left(t_{k, 2}\right)= & \hat{x}\left(t_{k, 2}^{-}\right)-H\left(\hat{y}\left(t_{k, 2}^{-}\right)-y_{q}\left(t_{k, 2}\right)\right. \\
& \left.t_{k, 2}=k T ; \quad-\operatorname{Sat}_{\frac{\Delta}{2}}\left(\hat{y}\left(t_{k, 2}^{-}\right)-y_{q}\left(t_{k, 2}\right)\right)\right), \\
\hat{y}(t)= & C_{r} \hat{x}(t)
\end{aligned}
$$

where $\hat{x}\left(t_{k, 1}\right)$ and $\hat{x}\left(t_{k, 2}\right)$ are the new estimated state at time $t_{k, 1}, t_{k, 2}$, respectively.

$$
\hat{y}\left(t_{k, 2}\right)= \begin{cases}y_{q}\left(t_{k, 2}\right)+\operatorname{Sat}_{\frac{\Delta}{2}}\left(\hat{y}\left(t_{k, 2}^{-}\right)-y_{q}\left(t_{k, 2}\right)\right), & \text { if }\left|\hat{y}\left(t_{k, 2}^{-}\right)-y_{q}\left(t_{k, 2}\right)\right|>\frac{\Delta}{2} \\ \hat{y}\left(t_{k, 2}^{-}\right), & \text {otherwise }\end{cases}
$$




\section{B. Stability Analysis}

We now analyze the stability of the RKSE (25)-(28). Define the estimator error

$$
e(t) \triangleq x(t)-\hat{x}(t)
$$

Subtracting the estimator (25)-(27) from the kinematic model (13) yields the estimator error system with the following dynamic equations:

$$
\begin{aligned}
\dot{e}(t) & =A_{e} e(t)+B_{r} n_{a}(t)+L \varepsilon(t) \\
e(0) & =e_{0}, t \notin\left\{t_{k, 1} ; t_{k, 2}\right\} \\
e\left(t_{k, 1}\right) & =D e\left(t_{k, 1}^{-}\right) \\
e\left(t_{k, 2}\right) & =D e\left(t_{k, 2}^{-}\right)+H \psi\left(t_{k, 2}\right)
\end{aligned}
$$

where

$$
\begin{aligned}
A_{e} & =A_{r}-L C_{r} \\
D & =I-H C_{r} \\
\psi\left(t_{k, 2}\right) & =\varepsilon\left(t_{k, 2}\right)-\operatorname{Sat}_{\frac{\Delta}{2}}\left(\hat{y}\left(t_{k, 2}^{-}\right)-y_{q}\left(t_{k, 2}\right)\right) .
\end{aligned}
$$

Lemma 1: The estimator error system (30) is uniformly bounded-input bounded-state (UBIBS) stable. More specifically, for any $\alpha \geq 0$ and $\bar{n}_{a} \geq 0$, there exists $\mu>0$ such that

$$
\|e(0)\| \leq \alpha,\left|n_{a}(t)\right| \leq \bar{n}_{a}, \forall t \geq 0 \Rightarrow\|e(t)\| \leq \mu, \forall t \geq 0 .
$$

Proof: See Appendix.

\section{Simulated Comparison of RKSE and SSE}

We carry out simulation studies on the LM plant model to show the effectiveness of the reset actions in state estimation. The performance index compared is the root mean square (RMS) of the sampled position estimation error $e_{y}$, which is defined by

$$
\operatorname{RMS}\left(e_{y}\right)=\sqrt{\sum_{k=1}^{N} e_{y}^{2}(k) / N}
$$

where $e_{y}=y-\hat{y}$, and $N$ is the number of the samples.

According to (30), the estimator gain should be selected to balance the effects of the acceleration sensing noise and quantization noise on the estimation error. The estimator gain $L$ for the RKSE and SSE is tuned by pole placement method, which is chosen as

$$
L=\left[\begin{array}{ll}
4 \pi \zeta f_{n} & 4 \pi^{2} f_{n}^{2} / k_{y}
\end{array}\right]^{T}
$$

where $\zeta=0.707$, and $f_{n}$ (in $\mathrm{Hz}$ ) is referred to as the estimator bandwidth that is tuned iteratively to achieve the smallest $\operatorname{RMS}\left(e_{y}\right)$ in (32). The estimator gain (33) will result in the eigenvalues of the estimator system matrix $A_{e}$ to be placed at $\left(-\zeta f_{n} \pm f_{n} \sqrt{1-\zeta^{2}}\right) 2 \pi$.

During the simulation, the LM is assumed to rest at an initial position with

$$
y(0)=23 \mu \mathrm{m} .
$$

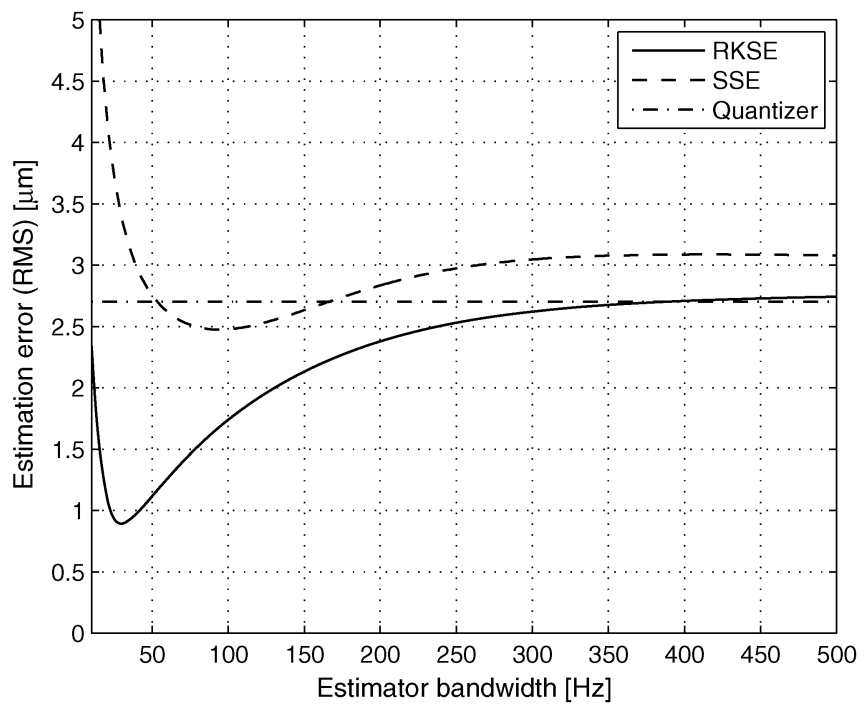

Fig. 8. Position estimation error versus estimator bandwidth $(r=50 \mu \mathrm{m})$.

A simple PID controller with the actual position as feedback is designed such that the position output $y$ follows a sinusoidal reference command

$$
y_{r}=r \cdot \operatorname{Sin}\left(2 \pi \omega_{0} t\right)
$$

where $\omega_{0}=10 \mathrm{~Hz}$, and $r$ (in micrometers) is the reference amplitude. The quantized position output $y_{q}$ together with the acceleration signals added by a white noise with a variance of 7.3 $\mathrm{m} / \mathrm{s}^{2}$ are then injected into the RKSE and SSE, respectively. The sampling period is set to be one cycle of the reference frequency, i.e., $0.1 \mathrm{~s}$. The reset interval for RKSE is $50 \mu$ s. The state estimators RKSE and SSE are run simultaneously.

Fig. 8 shows the simulation results of the achievable estimation errors versus estimator bandwidth for $r=50 \mu \mathrm{m}$. It is obvious that RKSE can achieve a significant smaller $\operatorname{RMS}\left(e_{y}\right)$ than that by either the SSE or the original quantizer. We can also see that a lower bandwidth (equivalent to smaller $L$ ) is allowed for RKSE to alleviate the quantization noise. The corresponding time traces of the position estimation are shown in Fig. 9, where one of the reset actions is marked, and it can be seen that RKSE has a faster transient convergence to the actual position and a smaller maximal estimation error in steady state. Finally, we carry out simulations with various reference magnitudes. The results are summarized in Table III. It can be seen from the columns that both the achievable smallest $\operatorname{RMS}\left(e_{y}\right)$ and $\max \left(\left|e_{y}\right|\right)$ in steady state by RKSE and SSE tend to decrease when $r$ increases. This is because the larger $r$ increases the rate of change of the position output, which in turn can be correctly updated by the quantizer more frequently and thus reduce the variance of the quantization noise. Hence, a higher bandwidth (larger $L$ ) is applied to suppress $n_{a}$. From the table, we see that the performance indices for the RKSE are the best in each case, which demonstrates that the RKSE is better at position estimation and more robust against reference amplitude changes. 

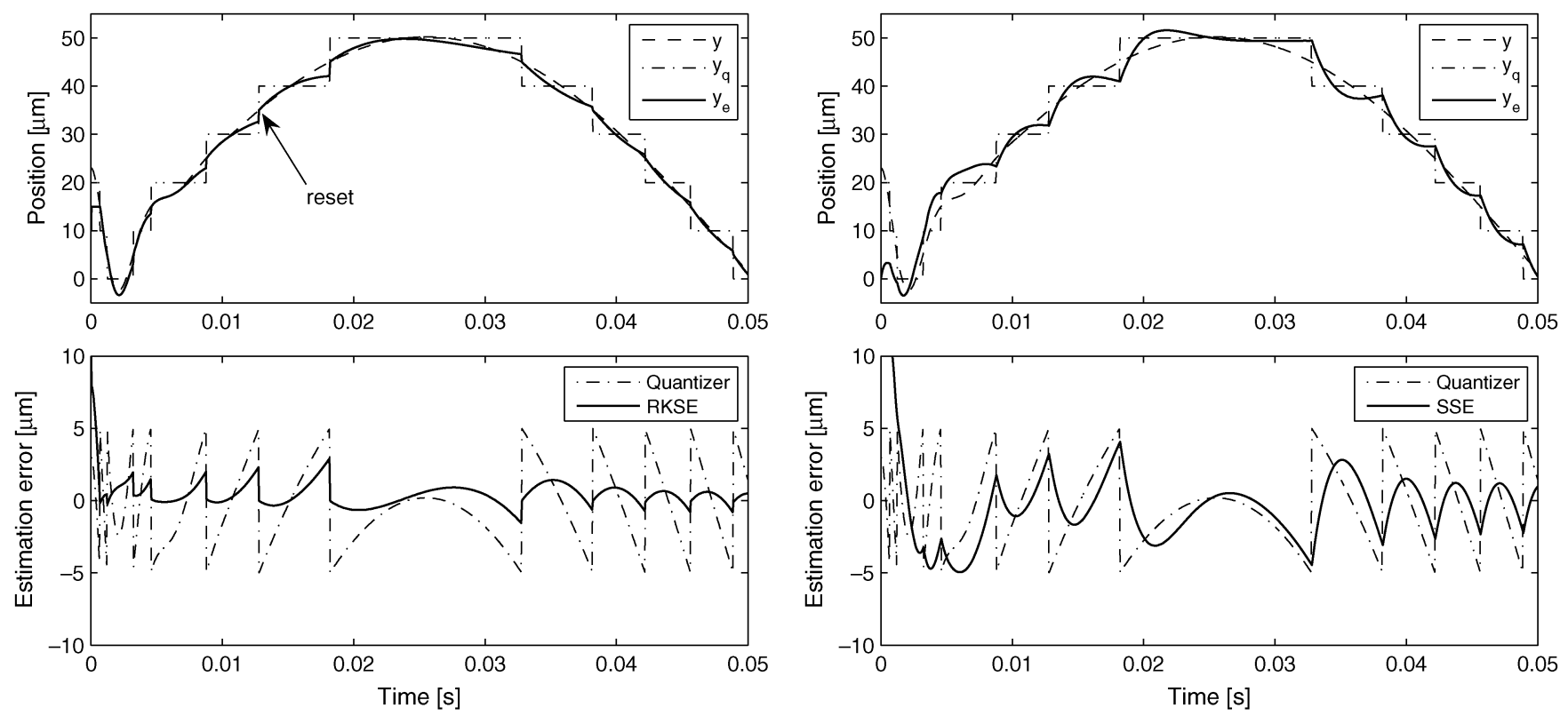

(a)

(b)

Fig. 9. Time responses of position estimation $(r=50 \mu \mathrm{m})$. (a) RKSE. (b) SSE.

\section{State FeEdBack Control Using IMP}

In sinusoidal reference tracking, a state feedback controller is designed to make the LM follow a sinusoidal reference command (35) with a known frequency. An effective design method is to use the IMP. This method includes the model of the reference signal as a part of the problem formulation and solving the problem of control in a tracking error space. The method is illustrated in detail in [33]. From Fig. 6, the nominal plant model is seen as (11), whose state-space representation is given by

$$
\begin{aligned}
& \dot{x}(t)=A x(t)+B u_{c}(t)+\Gamma w(t) \\
& y(t)=C x(t) \\
& \hat{x}(t)=x(t)-e(t)
\end{aligned}
$$

where

$$
\begin{aligned}
& x=\left[\begin{array}{l}
y \\
v
\end{array}\right], A=\left[\begin{array}{cc}
0 & k_{y} \\
0 & 0
\end{array}\right], B=\left[\begin{array}{c}
0 \\
\frac{k_{f}}{M}
\end{array}\right] \\
& \Gamma=\left[\begin{array}{l}
0 \\
1
\end{array}\right], C=\left[\begin{array}{ll}
1 & 0] .
\end{array}\right.
\end{aligned}
$$

Note that $\omega$ is the residual disturbance from the DOB as shown in (10), and $e$ is the estimation error (29).

For the purpose of control design, we first assume that the full state feedback is available and that $w(t)$ can be ignored. For the reference input (35), we then have the differential equation

$$
\ddot{y}_{r}+\omega_{0}^{2} y_{r}=0 .
$$

Define the tracking error as

$$
e_{t}=y_{r}-y \text {. }
$$

The problem of tracking $y_{r}$ can be seen as the regulation problem of tracking error, which means to design a control law such that the error $e_{t}$ tends to zero in the steady state. To do this, we define a new state and control input as follows:

$$
\begin{gathered}
\xi \triangleq \ddot{x}+\omega_{0}^{2} x \\
\mu \triangleq \ddot{u}_{c}+\omega_{0}^{2} u_{c}
\end{gathered}
$$

which relate the original plant state and control input to the differential equation of the reference (39). Accordingly, an overall system state equation can be described as

$$
\begin{aligned}
\dot{z} & =\left[\begin{array}{cc|c}
0 & 1 & 0_{1 \times 2} \\
-w_{0}^{2} & 0 & -C \\
\hline 0_{2 \times 1} & 0_{2 \times 1} & A
\end{array}\right] z+\left[\frac{0_{2 \times 1}}{B}\right] \mu \\
& \triangleq F z+G \mu
\end{aligned}
$$

where

$$
z \triangleq\left[e_{t} \dot{e}_{t} \xi\right]^{T} .
$$

It is easy to verify that $(F, G)$ is controllable, which implies that the error system (43) can have arbitrary closed-loop poles by state feedback. Therefore, there exists a control law

$$
\begin{aligned}
\mu & =-\left[\begin{array}{llll}
k_{1} & k_{2} & k_{3} & k_{4}
\end{array}\right] z \\
& \triangleq-K z
\end{aligned}
$$

such that the error system (43) has arbitrary pole placement. Expressing the control law in terms of $u_{c}$ and $x$ gives that

$$
u_{c}=-\frac{k_{2} s+k_{1}}{s^{2}+\omega_{0}^{2}} e-\left[k_{3} k_{4}\right] x
$$


TABLE III

COMPARISONS OF POSITION ESTIMATION PERFORMANCE

\begin{tabular}{|c|c|c|c|c|c|c|}
\hline \multirow{2}{*}{$\begin{array}{c}r \\
(\mu \mathrm{m})\end{array}$} & \multicolumn{2}{|c|}{ Achievable smallest RMS@bandwidth $(\mu \mathrm{m} @ \mathrm{~Hz})$} & \multicolumn{3}{|c|}{$\max \left(\left|e_{y}\right|\right)(\mu \mathrm{m})$} \\
\cline { 2 - 7 } & RKSE & SSE & Quantizer & RKSE & SSE & Quantizer \\
\hline 50 & $0.893 @ 30$ & $2.474 @ 94$ & 2.70 & 2.90 & 4.5 & 5 \\
100 & $0.792 @ 37$ & $1.995 @ 122$ & 2.748 & 2.44 & 4.4 & 5 \\
150 & $0.707 @ 39$ & $1.880 @ 146$ & 2.775 & 1.82 & 4.4 & 5 \\
200 & $0.702 @ 41$ & $1.762 @ 171$ & 2.763 & 1.48 & 4.3 & 5 \\
250 & $0.708 @ 44$ & $1.641 @ 192$ & 2.773 & 1.40 & 4.4 & 5 \\
\hline
\end{tabular}

The actual state is unavailable in practice, which should be replaced by the estimated state. Therefore, the real control input $u_{c}$ is given by

$$
u_{c}=\frac{k_{2} s+k_{1}}{s^{2}+\omega_{0}^{2}}\left(\hat{y}-y_{r}\right)-\left[k_{3} k_{4}\right] \hat{x}
$$

where $\hat{y}$ and $\hat{x}$ can be obtained from either the SSE or RKSE.

\section{A. System Analysis}

In real implementation of (48), the equivalent disturbance sources $\omega$ and $e$ will also enter the feedback loop. It is easy to derive the transfer function from the various inputs to the tracking error as follows:

$$
\begin{aligned}
e_{t}= & \frac{\left(s^{2}+\omega_{0}^{2}\right)\left(s^{2}+\frac{k_{f} k_{4}}{M} s-\frac{k_{f} k_{3} k_{y}}{M}\right)}{N(s)} y_{r}-\frac{k_{y}\left(s^{2}+\omega_{0}^{2}\right)}{N(s)} \omega \\
& +\frac{\frac{k_{f} k_{y}}{M}\left(s^{2}+\omega_{0}^{2}\right)}{N(s)}\left[k_{3} k_{4}\right] e-\frac{\frac{k_{f} k_{y}}{M}\left(k_{2} s+k_{1}\right)}{N(s)}\left[\begin{array}{ll}
1 & 0
\end{array}\right] e
\end{aligned}
$$

where

$N(s)=\left(s^{2}+\frac{k_{f} k_{4}}{M} s-\frac{k_{f} k_{3} k_{y}}{M}\right)\left(s^{2}+\omega_{0}^{2}\right)-\frac{k_{f} k_{y}}{M}\left(k_{2} s+k_{1}\right)$

whose roots are equivalent to the eigenvalues of $(s I-A+B K)$ that are designed to be stable in terms of (45). Therefore, we can see that the overall closed-loop system is stable and the tracking error $e_{t}$ is ensured to be bounded as a result of the bounded $\omega$ and $e$ that have been proved in previous sections. Especially, we have $\lim _{t \rightarrow \infty} e_{t}=0$ provided that both $\lim _{t \rightarrow \infty} \omega=0$ and $\lim _{t \rightarrow \infty} e=0$.

\section{EXPERIMENTAL RESULTS}

To show the effectiveness of the proposed control method, experiments are conducted on the LM positioning system. The advantages of the DOB and RKSE with accelerometer are demonstrated. For comparison, we also show the experimental results by a conventional DOB and SSE without accelerometer.

In the experiments, the same state feedback controller is used in all cases. The state feedback gain $K$ in (45) is designed such that the poles of the corresponding closed-loop system in (49) are placed at $[-88.8 \pm 88.9 i,-107 \pm 107 i]$. For the control scheme using accelerometer, the bandwidth of the filter $F_{d}(8)$ in the DOB is chosen as $600 \mathrm{~Hz}$, while the bandwidth of RKSE is chosen in terms of the reference amplitude and to minimize the RMS of tracking error. The bandwidth values for RKSE are found to be closed to the corresponding items in Table III. For the control scheme without accelerometer, the DOB is replaced by our previous design in [34] that uses the quantized position signals as input instead of the acceleration. In this DOB, we employ a third-order filter given by

$$
F_{d}(s)=\frac{3 \tau s+1}{(\tau s)^{3}+3(\tau s)^{2}+3 \tau s+1}
$$

where $\tau=0.0016$ corresponding to a frequency bandwidth of $100 \mathrm{~Hz}$, which has to be much lower than that with accelerometer for suppressing the quantization noise. Further, the SSE is used for state estimation with the control signals $u_{c}$ and quantized position as inputs. Similarly, we refer to the bandwidth values for SSE in Table III as initial values, which are then finely tuned to minimize the RMS of tracking error. Interestingly, we find that the bandwidth of SSE that achieves the smallest RMS of estimation error does not always lead to the smallest RMS of tracking error. This is mainly because the quantization noise in the case of sinusoidal position tracking is harmonic and nonstationary.

The overall controller was implemented on a real-time DSP system (dSPACE-DS1103, dSPACE GmbH, Paderborn, Germany) with the sampling period $0.2 \mathrm{~ms}$. After the LM control system was running steadily, we collected 2501 samples of the signals of interest, and then calculated the performance indices $\operatorname{RMS}\left(e_{t}\right), \max \left(e_{t}\right), \operatorname{RMS}(u), \max (u)$, respectively, where the RMS is defined similarly by (32). Figs. $10-12$ present the experimental results. Fig. 10 shows the time-domain responses of the position tracking, and from the top and bottom plots we can easily see that the actual position in (a) follows the reference command more accurately than that in (b). Moreover, the middle plots of Fig. 10 indicate that the control input in (a) is smoother and has less signal chattering than that in (b). We believe that the chattering is mainly caused by the quantization noise that enters the DOB without using accelerometer. This is because the chattering occurs at the points where the rate of change of the position is fast, which implies the corresponding quantization noise features frequent zero crossings at these points. This problem is largely amended when the accelerometer that offers smooth input signals is incorporated into the DOB. We also conducted experiments with various reference amplitudes to examine the robust tracking performance. Fig. 11 summaries the results for easy comparison. We can see that for any reference the performance indices from the experiments with accelerometer and RKSE (Exp A) outperform those without accelerometer and with SSE (Exp B). 

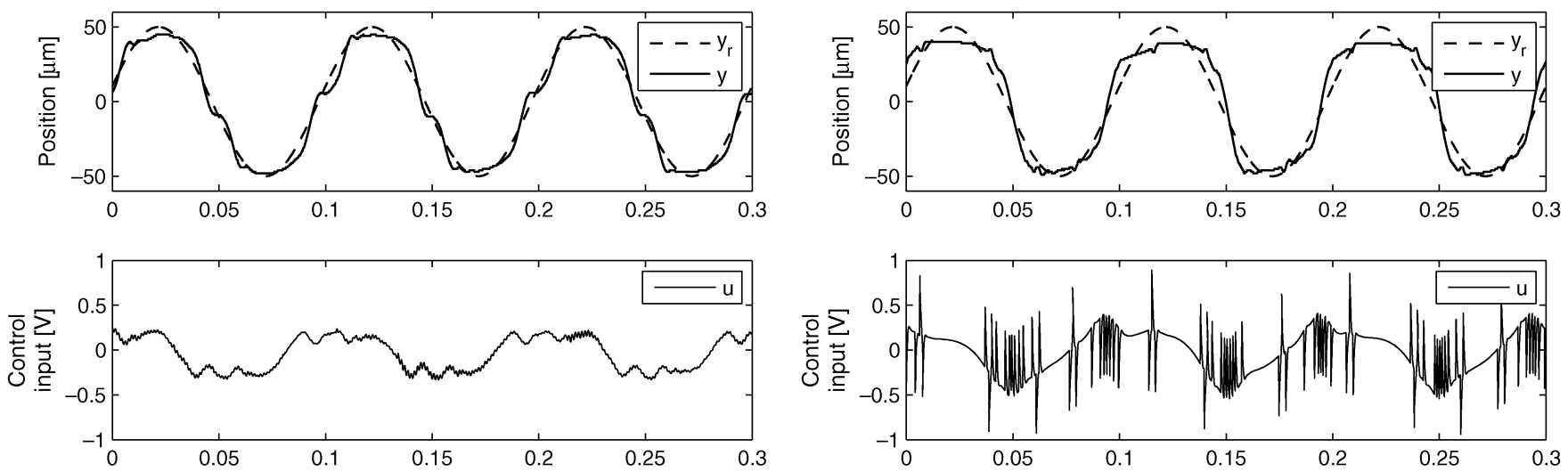

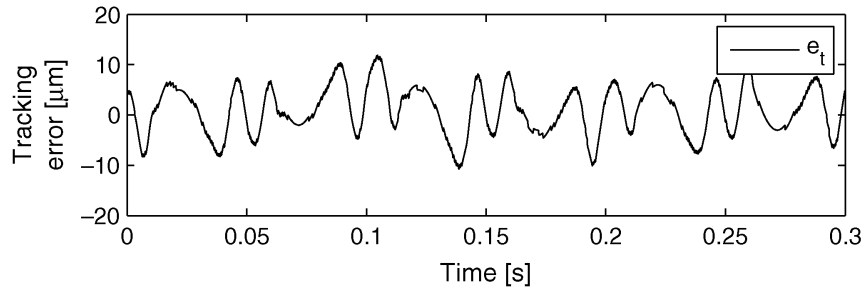

(a)

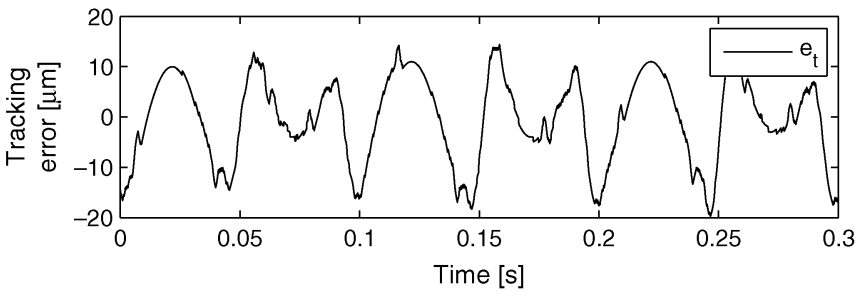

(b)

Fig. 10. Experimental position tracking performance $(r=50 \mu \mathrm{m})$. (a) With accelerometer and RKSE; (b) without accelerometer and with SSE.
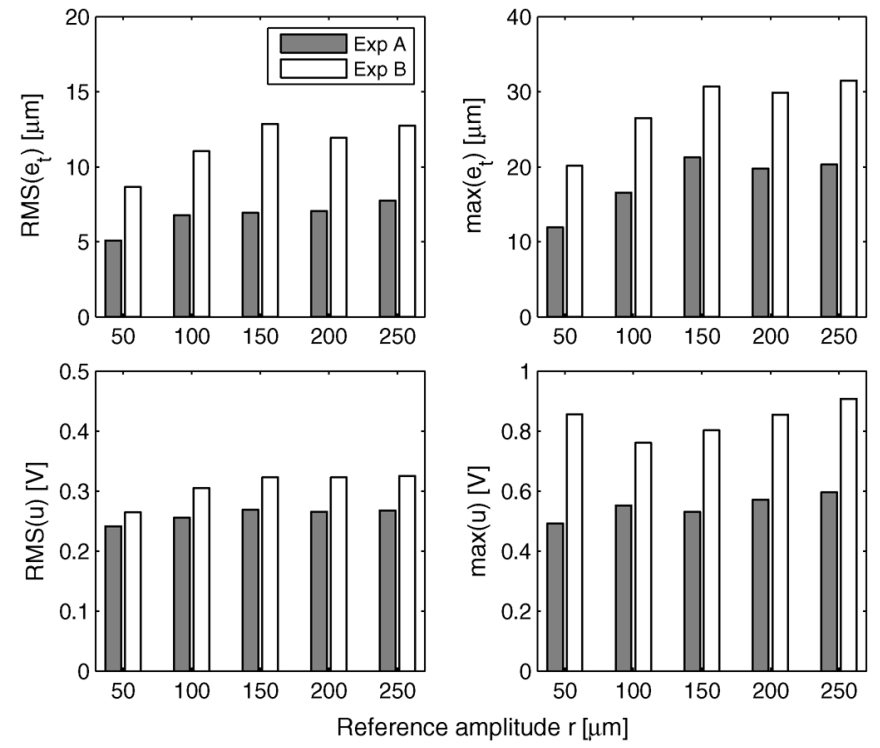

Fig. 11. Summary and comparison of experimental position tracking performance (Exp A indicates experiments with accelerometer and RKSE; Exp B indicates experiments without accelerometer and with SSE).

Finally, we evaluated the robust tracking performance when the LM stage was carrying a $1 \mathrm{~kg}$ payload. The results are shown in Fig. 12, which indicates that the controller with accelerometer and RKSE has better robustness in terms of smaller difference of $\operatorname{RMS}\left(e_{t}\right)$ between with payload and no payload. This verifies the effectiveness of the DOB on the compensation for gain variations.

In summary, we can see from the above results that the performance achieved by the setup with accelerometer and RKSE significantly outperforms that without accelerometer. From the

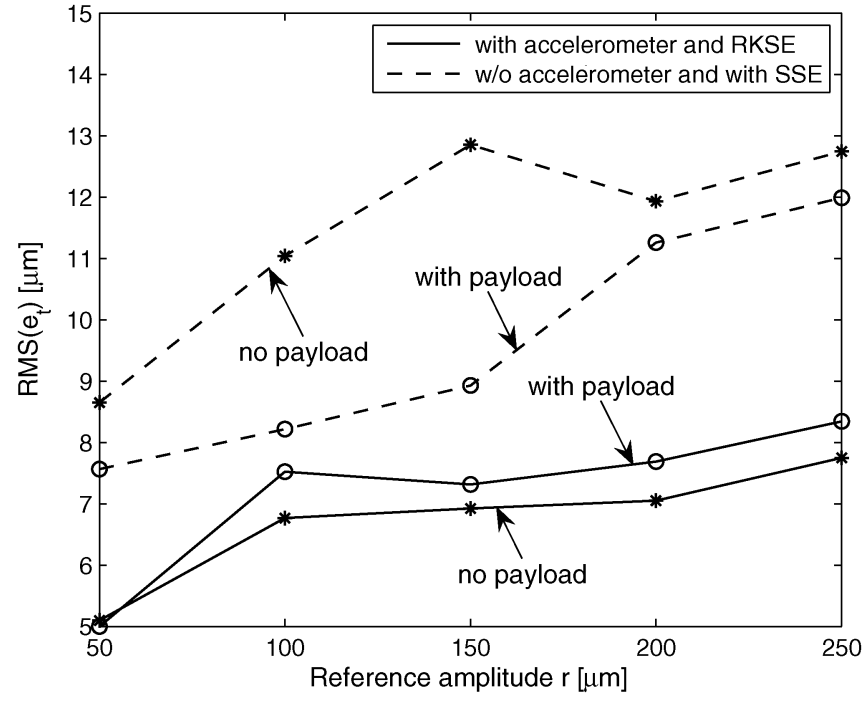

Fig. 12. Experimental robust tracking performance (payload $=1 \mathrm{~kg})$.

industrial point of view, the tradeoff between cost and performance is a critical issue. In our setup, the add-on accelerometer costs roughly $2 \%$ in the whole system budget. Alternatively, we may use a more expensive replacement encoder to achieve the equivalent improved tracking performance. From simulations, this requires an encoder of at least twice higher resolution than the original encoder. As far as we know from the manufacturer, such an encoder for our application will cost much more than an accelerometer. It should also be noted that the use of accelerometer can provide additional advantages such as control signal smoothing and enhanced robustness as shown in the above experimental results. Therefore, the scheme using the accelerometer and RKSE is cost-effective for motion control systems. 


\section{CONCLUSION}

This paper has attempted to enhance the tracking performance of an LM control system by incorporating a cost-effective accelerometer. The controller is composed of the DOB, the RKSE, and the IMP-based feedback controller, which are sequentially designed based on the fusion of the acceleration and quantized position signals. Particularly, the DOB is designed with the acceleration signals as feedback to prevent the quantization noise from entering the DOB loop. A RKSE is built with the acceleration signals as input that makes the state estimate immune to both system perturbations and input disturbances. Further, a reset technique is proposed in the RKSE, which can thus update the estimated state by using the extracted information from the quantizer output. Analysis and simulations have shown that the RKSE provides more accurate and robust position estimate than the SSE without reset. Lastly, we apply the IMP to design the state feedback controller for sinusoidal reference tracking. Experimental results have demonstrated that the use of accelerometer and RKSE can significantly enhance the position tracking performance and the robustness to system gain variations caused by payload.

The DOB and RKSE incorporating acceleration measurement is applicable in other motion control systems. However, it should be noted that other technical problems may arise for some specific applications. For example, in rotational motion control, the mounting of an accelerometer on the rotor is generally difficult and the transmitted signal from the rotor to the controller is easily influenced by electrical noise [21]. Further, for servo systems where the sampling rate is rather low or the encoder is significantly imperfect due to optical, mechanical and electrical inaccuracy [35], the performance of RKSE decreases because the trusted position transition is not captured accurately. To alleviate this problem, we may identify the imperfections by experiments and then precompensate for the imperfections prior to the RKSE. Finally, we may explore the design of the feedback controller and the estimator jointly to further suppress the quantization noise rather than individual design. We will further investigate these issues in future work.

\section{APPENDIX \\ PROOF OF LEMMA 1}

First, we consider the case of $t \notin\left\{t_{k, 1} ; t_{k, 2}\right\}$. Let $V(t)=$ $e(t)^{T} P e(t)$ be the Lyapunov function of the estimator error system (30). Thus, we have

$$
\begin{aligned}
\dot{V}(t)= & 2 e^{T} P \dot{e} \\
= & 2 e^{T} P\left(A_{e} e+B_{r} n_{a}+L \varepsilon\right) \\
= & 2 e^{T} P A_{e} e+2 e^{T} P^{\frac{1}{2}}\left(P^{\frac{1}{2}}\right)^{T}\left(B_{r} n_{a}+L \varepsilon\right) \\
\leq & -e^{T} e+\tau e^{T} P e+\frac{1}{\tau}\left(B_{r} n_{a}+L \varepsilon\right)^{T} P\left(B_{r} n_{a}+L \varepsilon\right) \\
\leq & e^{T}(-I+\tau P) e \\
& +\frac{1}{\tau}\left(B_{r} n_{a}+L \varepsilon\right)^{T} P\left(B_{r} n_{a}+L \varepsilon\right)
\end{aligned}
$$

where $\tau>0$ is a small scalar such that $-I+\tau P<0$. Since $w(t)$ and $\varepsilon(t)$ are both bounded, $V(t)$ is bounded.

Next, we evaluate the Lyapunov function increment at $t \in$ $\left\{t_{k, 1}\right\}$

$$
\begin{aligned}
\Delta V\left(t_{k, 1}\right) & =V\left(t_{k, 1}\right)-V\left(t_{k, 1}^{-}\right) \\
& =e\left(t_{k, 1}\right)^{T} \operatorname{Pe}\left(t_{k, 1}\right)-e\left(t_{k, 1}^{-}\right)^{T} P e\left(t_{k, 1}^{-}\right) \\
& =e\left(t_{k, 1}^{-}\right)^{T}\left(D^{T} P D-P\right) e\left(t_{k, 1}^{-}\right) \\
& =-e\left(t_{k, 1}^{-}\right)^{T}\left(C_{r}^{T}\left(C_{r} P^{-1} C_{r}^{T}\right)^{-1} C_{r}\right) e\left(t_{k, 1}^{-}\right) \\
& \leq 0 .
\end{aligned}
$$

Last, we evaluate the Lyapunov function increment at $t \in$ $\left\{t_{k, 2}\right\}$

$$
\begin{aligned}
\Delta V\left(t_{k, 2}\right)= & V\left(t_{k, 2}\right)-V\left(t_{k, 2}^{-}\right) \\
= & e\left(t_{k, 2}\right)^{T} P e\left(t_{k, 2}\right)-e\left(t_{k, 2}^{-}\right)^{T} P e\left(t_{k, 2}^{-}\right) \\
= & e\left(t_{k, 2}^{-}\right)^{T}\left(D^{T} P D-P\right) e\left(t_{k, 2}^{-}\right) \\
& +\psi\left(t_{k, 2}\right)^{T} H^{T} P H \psi\left(t_{k, 2}\right) \\
= & -e\left(t_{k, 2}^{-}\right)^{T}\left(C_{r}^{T}\left(C_{r} P^{-1} C_{r}^{T}\right)^{-1} C_{r}\right) e\left(t_{k, 2}^{-}\right) \\
& +\psi\left(t_{k, 2}\right)^{T}\left(C_{r} P^{-1} C_{r}^{T}\right)^{-1} \psi\left(t_{k, 2}\right) \\
= & -\left(C_{r} P^{-1} C_{r}^{T}\right)^{-1} \\
& \times\left(\left(y\left(t_{k, 2}\right)-\hat{y}\left(t_{k, 2}^{-}\right)\right)^{2}-\psi\left(t_{k, 2}\right)^{2}\right) \\
= & -\left(C_{r} P^{-1} C_{r}^{T}\right)^{-1} \\
& \times\left(\left(y\left(t_{k, 2}\right)-\hat{y}\left(t_{k, 2}^{-}\right)-\psi\left(t_{k, 2}\right)\right)^{2}+2 \Gamma\right)
\end{aligned}
$$

where

$$
\Gamma=\left(\hat{y}\left(t_{k, 2}^{-}\right)-\hat{y}\left(t_{k, 2}\right)\right)\left(\hat{y}\left(t_{k, 2}\right)-y\left(t_{k, 2}\right)\right) .
$$

From (24), it is implied that

$$
\begin{gathered}
y\left(t_{k, 2}\right)<\hat{y}\left(t_{k, 2}\right)<\hat{y}\left(t_{k, 2}^{-}\right), \text {if } \hat{y}\left(t_{k, 2}^{-}\right)>y_{q}\left(t_{k, 2}\right)+\frac{\Delta}{2} \\
\hat{y}\left(t_{k, 2}^{-}\right)<\hat{y}\left(t_{k, 2}\right)<y\left(t_{k, 2}\right), \text { if } \hat{y}\left(t_{k, 2}^{-}\right)<y_{q}\left(t_{k, 2}\right)-\frac{\Delta}{2} .
\end{gathered}
$$

Accordingly, we have $\Gamma>0$, and thus

$$
\Delta V\left(t_{k, 2}\right) \leq 0 .
$$

Following (52), we can show that there exist positive $c_{0}, c_{1}$, and $c_{2}$ such that

$$
\|e(t)\|^{2} \leq c_{0} e^{-\eta t}\|e(0)\|^{2}+c_{1} \bar{n}_{a}^{2}+c_{2} \Delta^{2}, \quad \forall t \geq 0
$$

where $\eta=\lambda_{\min }(I-\tau P) / \lambda_{\max }(P)$. We can thus take

$$
\mu=\sqrt{c_{0} \alpha^{2}+c_{1} \bar{n}_{a}^{2}+c_{2} \Delta^{2}}
$$

for (31) 


\section{REFERENCES}

[1] R. Lorenz and K. Van Patten, "High resolution velocity estimation for all digital, ac servo drives," IEEE Trans. Ind. Appl., vol. 27, no. 4, pp. 701-705, Jul./Aug. 1991.

[2] J. Bertram, "The effect of quantization in sampled-feedback systems," Trans. AIEE, vol. 77, pt. 2, pp. 177-182, Sep. 1958.

[3] G. F. Franklin, J. D. Powell, and M. Workman, Digital Control of Dynamic Systems, 3rd ed. Reading, MA: Addison-Wesley, 1998.

[4] B. Widrow, I. Kollar, and M. Liu, "Statistical theory of quantization," IEEE Trans. Autom. Control, vol. 45, no. 2, pp. 353-361, Apr. 1996.

[5] R. Kavanagh and J. Murphy, "The effects of quantization noise and sensor nonideality on digital differentiator-based rate measurement," IEEE Trans. Instrum. Meas., vol. 47, no. 6, pp. 1457-1463, Dec. 1998.

[6] D. Luong-Van, M. Tordon, and J. Katupitiya, "Covariance profiling for an adaptive Kalman filter to suppress sensor quantization effects," in Proc. IEEE Conf. Decision Control, 2004, pp. 2680-2685.

[7] S. Chang and M. Perng, "State estimation from incremental sensor data corrupted by track miscounts and a detection delay," IEEE Trans. Control Syst. Technol., vol. 4, no. 1, pp. 65-71, Jan. 1996.

[8] B. D. O. Anderson and J. B. Moore, Optimal Filtering. Englewood Cliffs, NJ: Prentice-Hall, 1979.

[9] S. Julier and J. Uhlmann, "A new extension of the Kalman filter to nonlinear systems," in Proc. AeroSense: 11th Int. Symp. Aerosp./Def. Sens., Simulation Controls, 1997, pp. 182-193.

[10] M. Arulampalam, S. Maskell, N. Gordon, and T. Clapp, "A tutorial on particle filters for online nonlinear/non-Gaussian Bayesian tracking," IEEE Trans. Signal Process., vol. 50, no. 2, pp. 174-188, Feb. 2002.

[11] F. Gustafsson, F. Gunnarsson, N. Bergman, U. Forssell, J. Jansson, R. Karlsson, and P. Nordlund, "Particle filters for positioning, navigation, and tracking," IEEE Trans. Signal Process., vol. 50, no. 2, pp. 425-437, Feb. 2002

[12] F. Daum, "Nonlinear filters: Beyond the Kalman filter," IEEE AES Syst. Mag., vol. 20, no. 8, pp. 57-69, Aug. 2005.

[13] A. Hodel and Y. Hung, "A state estimator with reduced sensitivity to sensor quantization," in Proc. Annu. Conf. IEEE Ind. Electron. Soc., 2003, pp. 586-590.

[14] E. Sviestins and T. Wigren, "Optimal recursive state estimation with quantized measurements," IEEE Trans. Autom. Control, vol. 45, no. 4, pp. 762-767, Apr. 2000.

[15] J. Sur and B. Paden, "State observer for linear time-invariant systems with quantized output," J. Dyn. Syst., Meas., Control, vol. 120, pp. 423-426, Sep. 1998.

[16] D. Delchamps, "Extracting state information from a quantized output record," Syst. Control Lett., vol. 13, no. 5, pp. 365-372, Dec. 1989.

[17] M. White and M. Tomizuka, "Increased disturbance rejection in magnetic disk drives by acceleration feedforward control and parameter adaption," Control Eng. Practice, vol. 5, no. 6, pp. 741-751, Jun. 1997.

[18] J. Ishikawa and M. Tomizuka, "Pivot friction compensation using an accelerometer and a disturbance observer for hard disk drives," IEEE/ ASME Trans. Mechatron., vol. 3, no. 3, pp. 194-201, Sep. 1998.

[19] K. Tan, S. Lim, T. Lee, and H. Dou, "High-precision control of linear actuators incorporating acceleration sensing," Robot. Comput. Integr. Manuf., vol. 16, no. 5, pp. 295-305, Oct. 2000.

[20] H. Shim, M. Kochem, and M. Tomizuka, "Use of accelerometer for precision motion control of linear motor driven positioning system," in Proc. Annu. Conf. IEEE Ind. Electron. Soc., 1998, pp. 2409-2414.

[21] S. Jeon and M. Tomizuka, "Benefits of acceleration measurement in velocity estimation and motion control," Control Eng. Practice, vol. 15, no. 3, pp. 325-332, Mar. 2007.

[22] O. Beker, C. Hollot, Y. Chait, and H. Han, "Fundamental properties of reset control systems," Automatica, vol. 40, no. 6, pp. 905-915, Jun. 2004.

[23] Y. Guo, Y. Wang, J. Zheng, and L. Xie, "Stability analysis, design and application of reset control systems," in Proc. IEEE Conf. Control Autom., 2007, pp. 3196-3201.

[24] G. F. Franklin, J. D. Powell, and A. Emami-Naeini, Feedback Control of Dynamic Systems, 3rd ed. Reading, MA: Addison-Wesley, 1994.

[25] J. Zheng and M. Fu, "A reset state estimator for linear systems to suppress sensor quantization effects," in Proc. 17th IFAC World Congr., 2008, pp. 9254-9259.
[26] E. Papadopoulos and G. Chasparis, "Analysis and model-based control of servomechanisms with friction," in Proc. IEEE/RSJ Int. Conf. Intell. Robots Syst., 2002, pp. 2109-2114.

[27] A. Radke and Z. Gao, "A survey of state and disturbance observers for practitioners," in Proc. Amer. Control Conf., 2006, pp. 5183-5188.

[28] M. White, M. Tomizuka, and C. Smith, "Improved track following in magnetic disk drives using a disturbance observer," IEEE/ASME Trans. Mechatron., vol. 5, no. 1, pp. 3-11, Mar. 2000.

[29] L. Yi and M. Tomizuka, "Two-degree-of-freedom control with robust feedback control for hard disk servo systems," IEEE/ASME Trans. Mechatron., vol. 4, no. 1, pp. 17-24, Mar. 1999.

[30] K. Yang, Y. Choi, and W. Chung, "On the tracking performance improvement of optical disk drive servo systems using error-based disturbance observer," IEEE Trans. Ind. Electron., vol. 52, no. 1, pp. 270-279, Feb. 2005.

[31] S. Katsura, Y. Matsumoto, and K. Ohnishi, "Modeling of force sensing and validation of disturbance observer for force control," IEEE Trans. Ind. Electron., vol. 54, no. 1, pp. 530-538, Feb. 2007.

[32] K. Natori and K. Ohnishi, "A design method of communication disturbance observer for time-delay compensation, taking the dynamics property of network disturbance into account," IEEE Trans. Ind. Electron., vol. 55, no. 5, pp. 2152-2168, May 2008.

[33] F. Lewis, Applied Optimal Control and Estimation, Digital Design and Implementation. Englewood Cliffs, NJ: Prentice-Hall, 1992.

[34] J. Zheng and M. Fu, "Nonlinear feedback control of a dual-stage actuator system for reduced settling time," IEEE Trans. Control Syst. Technol., vol. 16, no. 4, pp. 717-725, Jul. 2008.

[35] C. Yien, "Incremental encoder error: Causes and ways to reduce them," in Proc. Int. Incremental Motion Conf., 1992, pp. 110-121.

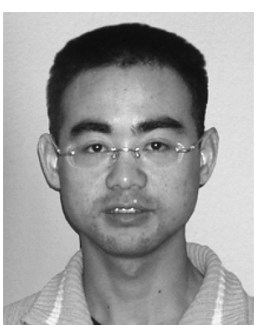

Jinchuan Zheng received the B.Eng. and M.Eng. degrees in mechatronics engineering from Shanghai Jiao Tong University, Shanghai, China, in 1999 and 2002, respectively, and the Ph.D. degree in electrical engineering from Nanyang Technological University, Singapore, in 2006.

In 2005, he joined the ARC Centre of Excellence for Complex Dynamic Systems and Control, School of Electrical and Computer Engineering, The University of Newcastle, Callaghan, Australia, as a Research Academic. His research interests include nanopositioning system design, vibration analysis, sensing and control, and advanced control applications to mechatronics.

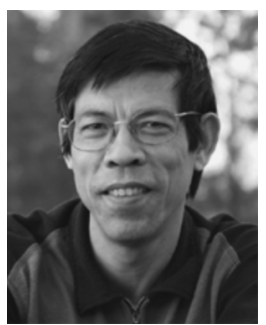

Minyue Fu (S'84-M'87-SM'94-F'02) received the Bachelor's degree in electrical engineering from the University of Science and Technology of China, Hefei, China, in 1982, and the M.S. and Ph.D. degrees in electrical engineering from the University of Wisconsin-Madison, in 1983 and 1987, respectively.

$\mathrm{He}$ joined the Department of Electrical and Computer Engineering, the University of Newcastle, Callaghan, Australia, in 1989, where he has been the Head of Department and Head of School. Currently, he is a Chair Professor in Electrical Engineering. From 1987 to 1989, he served as an Assistant Professor in the Department of Electrical and Computer Engineering, Wayne State University, Detroit, MI. In addition, he was a Visiting Associate Professor with University of Iowa in 1995-1996, and a Senior Fellow/Visiting Professor at Nanyang Technological University, Singapore, 2002. His main research interests include control systems, signal processing and communications.

Dr. Fu has been an Associate Editor for the IEEE TRANSACTIONS ON Automatic Control, Automatica, and the Journal of Optimization and Engineering. 